\title{
Electron spectrum in confined cylindrical nanoheterosystem with finite depth of potential well
}

\author{
V.A.Holovatsky, O.M. Voitsekhivska, M.J.Mikhalyova, \\ M.M.Tkach \\ Chernivtsi National University, \\ 2 Kotsiubinsky Str., 274012 Chernivtsi, Ukraine
}

Received November 9, 2000

\begin{abstract}
Electron spectrum in cylindrical quantum dot $\mathrm{HgS}$ embedded into $\mathrm{ZnS}$ medium is calculated using the variational method with variational parameter in Hamiltonian. The dependence of energy spectrum on the quantum well sizes is established. The electron spectrum calculated in the framework of infinitely deep potential well is compared to the one obtained within the variational method. It is shown that the first method gives satisfactory results for the ground level only and at rather big sizes of quantum well.
\end{abstract}

Key words: electron, quantum dot, nanocrystal

PACS: $79.60 . j \mathrm{~V}$

\section{Introduction}

The progressive development in physics of $2 \mathrm{D}$ heterosystems with quantum wells and their practical utilization in semiconductor lasers attracts many scientists to investigate the systems with even smaller dimension, namely: quantum wires and quantum dots. In quantum dots (QD) - "artificial atoms", the charge carriers are confined in all three directions and have the totally discrete energy spectra. Since the lasers produced on the basis of QD would have the superhigh temperature stability of threshold density of current and generational frequency, small time of ground level occupation, and, consequently, the high working frequencies [1-3].

Several methods of QD creation have been known so far. The molecular-beam epitaxy method is used to produce complicated QD of spherical shape [4]. The transversal etching of quantum wires produces the cylindrical quantum dots. The pyramidal QD with a rectangular base are obtained in [3].

It is clear that the QD of spherical and cubic symmetry are the best theoretically investigated systems because when calculating the electron spectrum the Schrödinger equation has an exact solution for the potential well with finite depth 


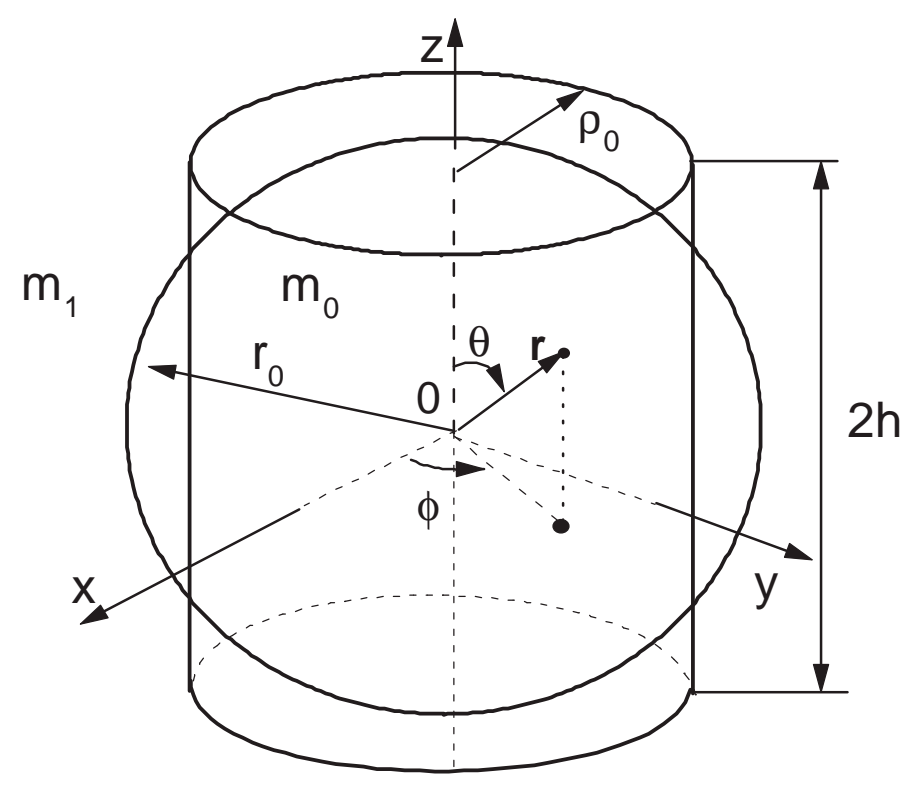

Figure 1. Geometry of cylinder approximated by sphere.

and infinite depth as well. The Schrödinger equation for the cylindrical quantum dot has an exact solution only in case of infinitely deep potential well in the direction of cylinder axis. For the QDs of a more complicated shape (pyramid, ellipse eth.) there are only the approximated methods of energy spectrum calculation. Therefore, the theory of quasiparticles (electron, hole, exciton, phonon) spectra is developed, generally, for the spatially confined spherical, cylindrical and rectangular heterosystems. Most of the scientists use the dielectric continuum model for the description of contacting media and effective mass approximation for the quasiparticles. The results of exciton spectra investigation in complicated spherical heterosystems [4] prove the possibility of applying these methods for QD of $30-40 \AA$ sizes.

In this paper the spectrum and wave functions of quasiparticle in cylindrical potential well are obtained within the Bethe variational method. Herein, the cylindrical potential well is approximated by a spherical well with the radius assumed as variational parameters. The numerical calculations are performed for the $\mathrm{HgS} / \mathrm{ZnS}$ heterosystem. The energy levels dependences on cylinder sizes are analysed.

\section{Hamiltonian of the system and solution of Schrödinger equa- tion in zeroth approximation}

The nanoheterosystem consisting of $\mathrm{HgS}$ semiconductor nanocrystal having the cylindrical shape with $\rho_{0}$ radius and $2 h$ height embedded into the $\mathrm{ZnS}$ semiconductor medium is under study. The beginning of the energy starts from the energy of the bottom of $\mathrm{ZnS}$ conductive band since an electron is moving inside the the potential well

$$
U(\vec{r})= \begin{cases}-V_{0} ; & 0 \leqslant \rho \leqslant \rho_{0},-h \leqslant z \leqslant h \\ 0 ; & \text { other region. }\end{cases}
$$


Thus, one has to solve the problem of quasiparticle energy spectrum and wave functions in rectangular potential well which has the shape of cylinder with finite height.

The Schrödinger equation has the form

$$
\left(\frac{-\hbar^{2}}{2} \vec{\nabla} \frac{1}{m(\vec{r})} \vec{\nabla}+U(\vec{r})\right) \psi(\vec{r})=E \psi(\vec{r})
$$

where

$$
m(\vec{r})= \begin{cases}m_{0} ; & 0 \leqslant \rho \leqslant \rho_{0},-h \leqslant z \leqslant h, \\ m_{1} ; & \text { other region, }\end{cases}
$$

Equation (2) with the potential (1) and masses (3) cannot be solved exactly. Therefore, using the Bethe variational method, cylinder is approximated by a sphere with $r_{0}$ radius, playing the role of the variational parameter. The approximated potential is written as

$$
V_{s}(r)= \begin{cases}-V_{0} ; & 0 \leqslant r \leqslant r_{0} \\ 0 ; & r_{0}<r<\infty\end{cases}
$$

and approximated mass

$$
m_{s}(r)= \begin{cases}m_{0} ; & 0 \leqslant r \leqslant r_{0} \\ m_{1} ; & r_{0}<r<\infty\end{cases}
$$

The Hamiltonian of the system is given as

$$
H=H_{0}+\Delta H,
$$

where

$$
H_{0}=-\frac{\hbar^{2}}{2} \vec{\nabla} \frac{1}{m_{s}(r)} \vec{\nabla}+V_{s}(r)
$$

is the electron Hamiltonian in approximated spherical heterosystem,

$$
\Delta H=-\frac{\hbar^{2}}{2} \vec{\nabla} \frac{1}{m(\vec{r})} \vec{\nabla}+\frac{\hbar^{2}}{2} \vec{\nabla} \frac{1}{m_{s}(r)} \vec{\nabla}+U(\vec{r})-V_{s}(r)
$$

is the Hamiltonian of perturbation.

It is clear that the energy states in cylindrical quantum well must be located in the interval between the corresponding states in inscribed and described spherical quantum wells. Thus, the Hamiltonian of quasiparticle is assumed as a basic part of $H_{0}$ Hamiltonian in spherical potential well with certain $r_{0}$ radius the magnitude of which would be fixed, according to the Bethe variational method, from the functional of the total energy. The Schrödinger equation with $H_{0}$ is solved exactly in the spherical coordinate system and has the form

$$
H_{0}(r, \vartheta, \phi) \Psi(r, \vartheta, \phi)=E_{0} \Psi(r, \vartheta, \phi) .
$$

Its solution is written as

$$
\Psi_{n l m}(r, \vartheta, \phi)=R_{n l}(r) Y_{l m}(\vartheta, \phi)
$$


The radial functions are given by

$$
R_{n l}(r)= \begin{cases}R_{n l}^{(0)}(r)=A_{0} j_{l}\left(k_{n} r\right) ; & r \leqslant r_{0}, \\ R_{n l}^{(1)}(r)=A_{1} h_{l}^{(+)}\left(i \chi_{n} r\right) ; & r>r_{0}\end{cases}
$$

where

$$
k=\sqrt{\frac{2 m_{0}\left(V_{0}-|E|\right)}{\hbar^{2}}}, \quad \chi=\sqrt{\frac{2 m_{1}|E|}{\hbar^{2}}} .
$$

In the media interface $\left(r=r_{0}\right)$ the following conditions should be satisfied

$$
\left\{\begin{array}{l}
\left.R_{n l}^{0}\left(r_{0}\right)\right|_{r=r_{0}}=\left.R_{n l}^{1}\left(r_{0}\right)\right|_{r=r_{0}}, \\
\left.\frac{1}{m_{0}} \frac{\mathrm{d} R_{n l}^{0}}{\mathrm{~d} r}\right|_{r=r_{0}}=\left.\frac{1}{m_{1}} \frac{\mathrm{d} R_{n l}^{1}}{\mathrm{~d} r}\right|_{r=r_{0}} .
\end{array}\right.
$$

From (13) one can get the dispersion equation for defining the quasiparticle energy spectrum

$$
\frac{1}{m_{0}}\left(l-k_{n}^{0} r_{0} \frac{j_{l+1}\left(k_{n}^{0} r_{0}\right)}{j_{l}\left(k_{n}^{0} r_{0}\right)}\right)=\frac{1}{m_{1}}\left(l-i \chi_{n}^{1} r_{0} \frac{h_{l+1}^{+}\left(i \chi_{n}^{1} r_{0}\right)}{h_{l}^{+}\left(i \chi_{n}^{1} r_{0}\right)}\right) .
$$

Coefficients $A_{1}$ and $A_{0}$ have the relationship

$$
A_{1}=A_{0} \frac{j_{l}\left(k_{n} r_{0}\right)}{h_{l}^{+}\left(i \chi_{n} r_{0}\right)}
$$

In the case $l=0$, equation (14) has the form

$$
1+\chi_{n}^{1} r_{0}-\frac{m_{1}}{m_{0}}\left(1-k_{n}^{0} r_{0} \cot \left(k_{n}^{0} r_{0}\right)\right)=0 .
$$

The $r_{0}$ variational parameter is found in the framework of variational method from the minimum condition of the functional built from the basic Hamiltonian $H_{0}$ and perturbation $\Delta H$ averaged on the wave functions (11).

\section{Calculation of non-spherical correction}

The electron energy spectrum in the first approximation is defined as a solution of zeroth problem obtained from dispersion equation (14) plus the correction found on the wave functions of the previous section. Thus

$$
E_{n l m}\left(r_{0}\right)=\epsilon_{n l}\left(r_{0}\right)+<n l m|\Delta \hat{H}| n l m>.
$$

The correction to the basic Hamiltonian is convenient to write as

$$
\Delta \hat{H}=\Delta \hat{H}_{0}+\Delta \hat{H}_{1}
$$


where $\Delta H_{0}=T_{0}+U_{0}$ is the Hamiltonian operating to the wave function in space inside the sphere with $r_{0}$ radius but outside the cylinder $\left(\Omega_{0}\right) ; \Delta H_{1}=T_{1}+U_{1}$ is the Hamiltonian operating to the wave function in space outside the sphere but inside the cylinder $\left(\Omega_{1}\right)$. From equation (8) there are

$$
\begin{aligned}
& \Delta \hat{H}_{0}=\Theta_{0}(\vec{r})\left\{\frac{\hbar^{2}}{2}\left(\frac{1}{m_{1}}-\frac{1}{m_{0}}\right)\left[\frac{\mathrm{d}^{2}}{\mathrm{~d} r^{2}}-\frac{l(l+1)}{r^{2}}\right]+V_{0}\right\}, \\
& \Delta \hat{H}_{1}=\Theta_{1}(\vec{r})\left\{\frac{\hbar^{2}}{2}\left(\frac{1}{m_{0}}-\frac{1}{m_{1}}\right)\left[\frac{\mathrm{d}^{2}}{\mathrm{~d} r^{2}}-\frac{l(l+1)}{r^{2}}\right]-V_{0}\right\},
\end{aligned}
$$

where $\Theta_{0}(\vec{r})$ and $\Theta_{1}(\vec{r})$ functions define the region of operation of corresponding operators and have the form

$$
\Theta_{0(1)}(\vec{r})= \begin{cases}1 ; & \vec{r} \in \Omega_{0(1)}, \\ 0 ; & \vec{r} \notin \Omega_{0(1)} .\end{cases}
$$

The wave functions (11) are the eigenfunctions of $\Delta H$ operator, then the functional (17) is transformed to the equation

$$
E_{n l m}\left(r_{0}\right)=\epsilon_{n l}\left(r_{0}\right)+\left\{\left(1-\frac{m_{0}}{m_{1}}\right) \epsilon_{n l}+\frac{m_{0}}{m_{1}} V_{0}\right\} I_{n l m}^{(0)}+\left\{\left(1-\frac{m_{1}}{m_{0}}\right) \epsilon_{n l}-V_{0}\right\} I_{n l m}^{(1)},
$$

with the following notations

$$
\begin{aligned}
& I_{n l m}^{(0)}=\iiint_{\Omega_{0}}\left|R_{n l}^{(0)}(r)\right|^{2}\left|Y_{l m}(\theta, \phi)\right|^{2} r^{2} \mathrm{~d} r \sin \theta \mathrm{d} \theta \mathrm{d} \phi, \\
& I_{n l m}^{(1)}=\iiint_{\Omega_{1}}\left|R_{n l}^{(1)}(r)\right|^{2}\left|Y_{l m}(\theta, \phi)\right|^{2} r^{2} \mathrm{~d} r \sin \theta \mathrm{d} \theta \mathrm{d} \phi .
\end{aligned}
$$

Taking into account that

$$
Y_{l m}(\theta, \phi)=N_{l m} P_{l}^{(m)}(\cos \theta) \mathrm{e}^{\mathrm{i} m \phi},
$$

where

$$
N_{l m}=\sqrt{\frac{(l-|m|) !(2 l+1)}{4 \pi(l+|m|) !}}
$$

integrals (23) and (24) can be rewritten as

$$
\begin{aligned}
& I_{n l m}^{(0)}=2 \frac{(l-|m|) !(2 l+1)}{(l+|m|) !} \iint_{S_{0}}\left|R_{n l}^{(0)}(r)\right|^{2} r^{2} \mathrm{~d} r\left|P_{l}^{(m)}\right|^{2} \sin \theta \mathrm{d} \theta, \\
& I_{n l m}^{(1)}=2 \frac{(l-|m|) !(2 l+1)}{(l+|m|) !} \iint_{S_{1}}\left|R_{n l}^{(1)}(r)\right|^{2} r^{2} \mathrm{~d} r\left|P_{l}^{(m)}\right|^{2} \sin \theta \mathrm{d} \theta,
\end{aligned}
$$

where the integrating regions $S_{0}$ and $S_{1}$ for the case $\rho<r_{0}<h$ are shown in figure 2 . From (27) and (28) one can see that the degeneration over the quantum number $m$ is taken off and the difference of energy levels with different $m$ characterizes the magnitude of nanostructure "non-spherical" shape. 


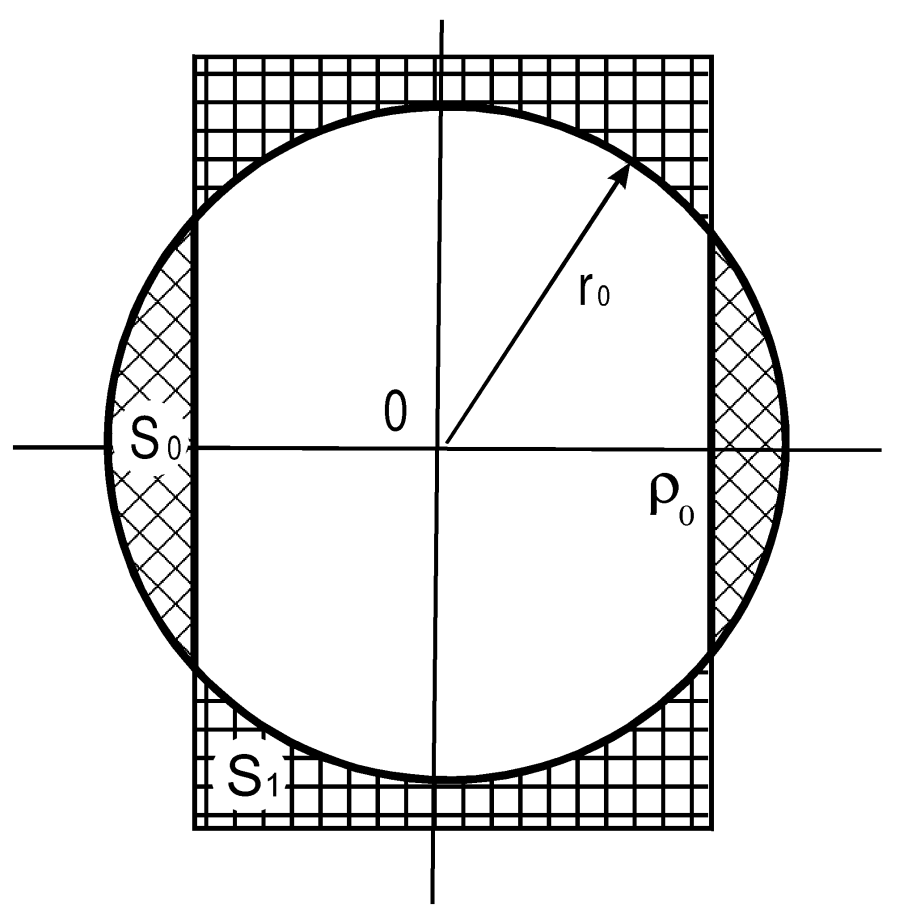

Figure 2. Scheme of integration regions $S_{0}$ and $S_{1}$ for the case $\rho_{0}<r_{0}<h$.

\section{Analysis of the results}

The calculation of electron spectrum in cylindrical nanoheterosystem $\mathrm{HgS} / \mathrm{ZnS}$ is performed using the formulas obtained in the previous sections. The crystals parameters are given in table 1 . The electron spectrum of the same system is calculated in the model of the infinitely deep potential well for comparison. It is well known that in this model the electron wave function is

$$
\Psi_{n n_{\rho} m}(\rho, \phi, z)=A J_{m}\left(\chi_{n_{\rho} m} \rho / \rho_{0}\right) f(z)
$$

where

$$
f(z)= \begin{cases}\sqrt{\frac{1}{h}} \cos \left(\frac{n \pi z}{2 h}\right) ; & n=1,3,5 \\ \sqrt{\frac{1}{h}} \sin \left(\frac{n \pi z}{2 h}\right) ; & n=2,4,6 .\end{cases}
$$

Table 1. Material parameters

\begin{tabular}{|c|c|c|c|}
\hline Material & $m_{e}$ & $a(\AA)$ & $V_{e}(\mathrm{eV})$ \\
\hline $\mathrm{ZnS}$ & 0.28 & 5.41 & -5.0 \\
\hline $\mathrm{HgS}$ & 0.036 & 5.85 & -3.1 \\
\hline
\end{tabular}




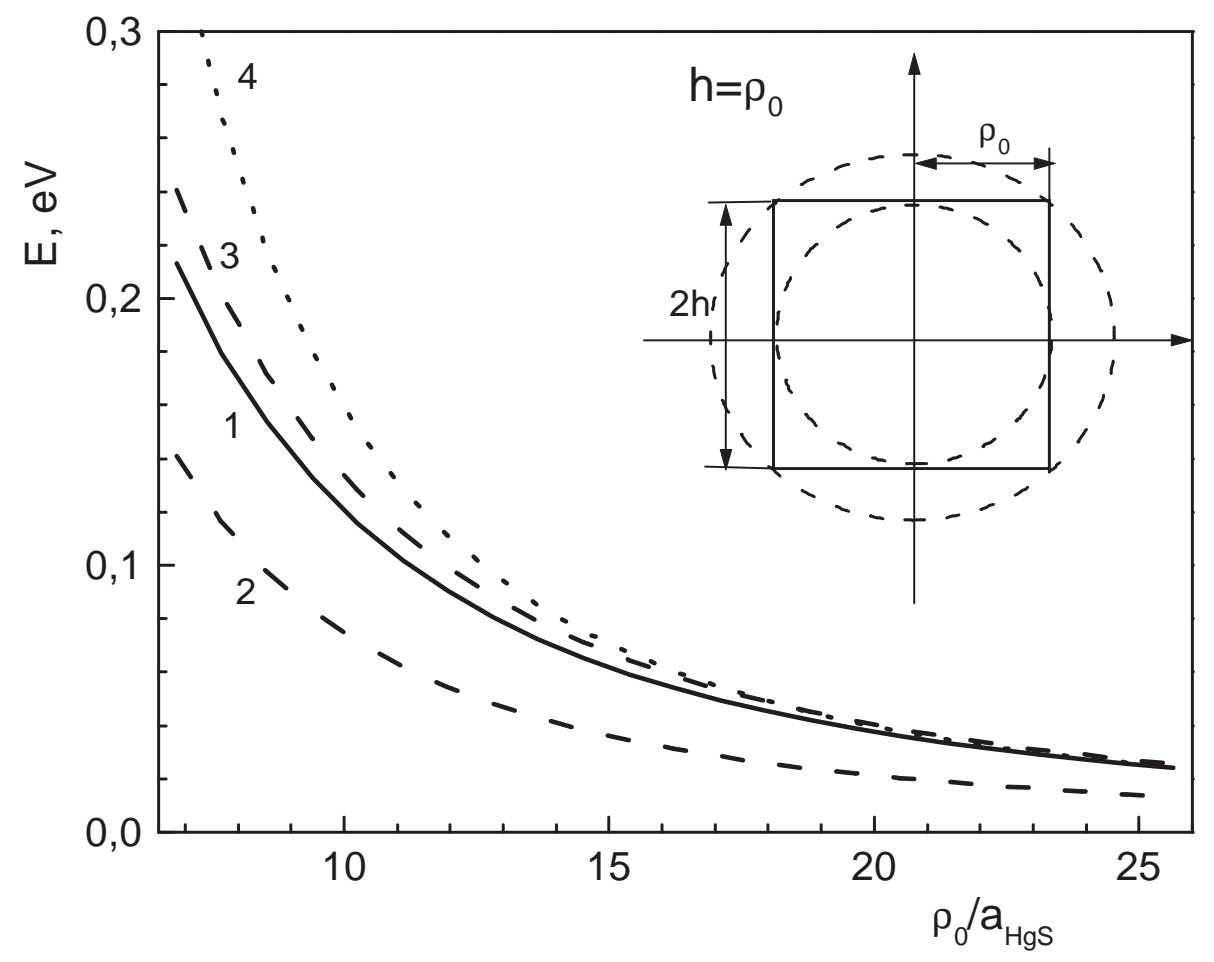

Figure 3. Dependence of electron ground state energy on cylinder radius: curve 1 - electron in cylindrical potential well with finite depth, 2 - electron in sphere describen around the cylinder, 3 - electron in the sphere inscriben into the cylinder, 4 - electron in infinitely deep cylinder.

The energy spectrum is defined from the equation

$$
E_{n_{\rho} m}=\frac{\hbar^{2}}{2 m_{0}}\left(\frac{\pi^{2} n^{2}}{4 h^{2}}+\frac{\xi_{n_{\rho} m}^{2}}{\rho_{0}^{2}}\right)
$$

where $\xi_{n_{\rho} m}$ - the zeros of Bessels functions $J_{m}\left(\xi_{n_{\rho} m}\right)$.

From physical considerations it is clear that the energy levels from the electron spectrum in a cylinder of finite sizes must be located between the corresponding energy levels of the electron in the sphere inscribed into the cylinder and described around it. Thus, the calculation and analysis of electron spectrum is performed for both spheres.

The dependence of energy levels on cylinder sizes was obtained at the condition $\rho_{0}=h$. The results are shown in figure 3 and figure 4 .

From the figures one can see the following features. All energy levels in all models are shifted to the bottom of the well when the quantum well is widening. At any well sizes, all energy levels in the model under research are really located between the respective levels of inscriben and describen spheres models. As far as the model of infinitely deep potential well is concerned, figure 3 proves that it gives satisfactory results for the ground level in the wells with a rather big size $\left(\rho_{0} \geqslant 15 a_{\mathrm{HgS}}\right)$. When $\rho_{0}<15 a_{\mathrm{HgS}}$ the unexactness of this model sharply increases. It is clear, because 


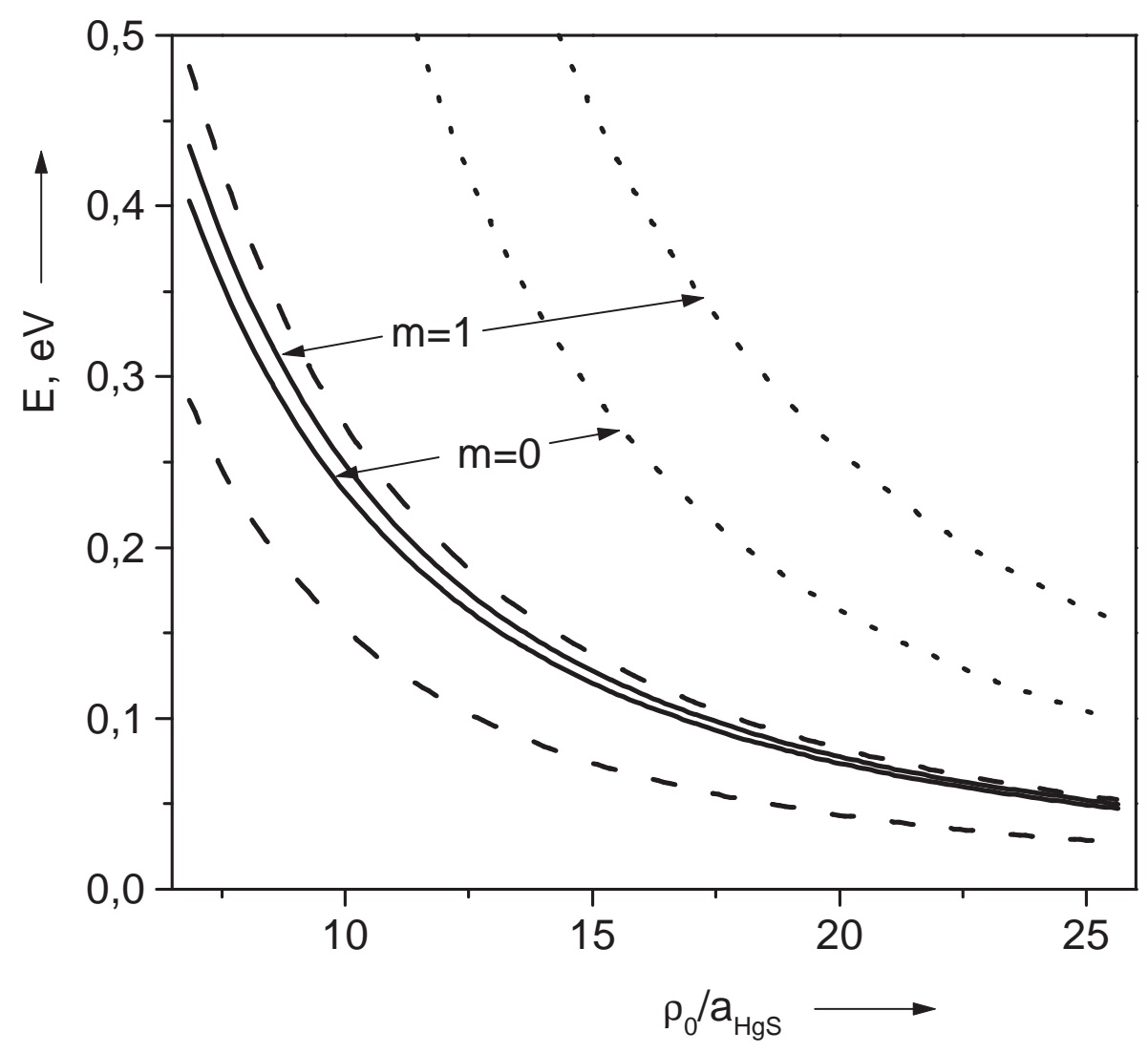

Figure 4. Energy of the electron in state $n=1, l=1$ as a function of cylinder radius: 1,2 - electron in cylindrical potential well with finite depth and quantum number $m=0,1$, respectively; 3(4) - electron in the sphere describen around (inscriben into) the cylinder; 5,6 - electron in infinitely deep cylinder with quantum number $m=0,1$, respectively.

when the height of the potential barrier is rather big $\left(V_{0}=V_{e}^{\mathrm{ZnS}}-V_{e}^{\mathrm{HgS}}=1,9 \mathrm{eV}\right.$, see table 1) the electron has a small probability to tunnel into $\mathrm{ZnS}$ medium and, thus, such states are well described by the model of infinitely deep potential well. When cylinder sizes become smaller, the energy levels shift into the region of higher energies and, thus, the probability of tunnelling into the external medium increases because the barrier effective height decreases. Due to these, the model of infinitely deep well is rough for the description of such states. Figure 4 proves that the excited states with $l=1$ are degenerated in both models with spheres. The degeneration is partly taken off for the model under research and for infinitely deep potential model. Herein it is clear that the latter one is extremely rough in the region of quantum dots characteristic sizes $(100 \div 150 \AA)$. The reason for such a situation was explained before. In the model under research both levels $(n=1, l=1, m=0$ and $n=1, l=1, m= \pm 1$ ) are located between the corresponding degenerated levels of inscriben and describen spheres and the magnitude of energy difference between states with $m= \pm 1$ and $m=0$ characterizes the "non-spherical" shape of the quantum dot. 
Finally, we must note that the presented method can be used for the approximated spectrum calculation for the quantum dots the geometric shape of which does not permit to obtain the exact solutions.

\title{
References
}

1. Alferov Zh.I. History and future of semiconductor heterostructures. // Fiz. Tehn. Pol., 1998, vol. 32, No. 1, p. 3-11 (in Russian).

2. Bimberg D. Quantum dots: paradigm changes in semiconductor physics. // Fiz. Tehn. Pol., 1998, vol. 33, No. 9, p. 1044-1048 (in Russian).

3. Schoos D., Mews A., Weller H. Quantum dot quantum well CdS/HgS/CdS. // Phys. Rev. B., 1994, vol. 49, No. 24, p. 17072-17078.

4. Ledentsov N.N., Ustinov V.M., Shchukin V.A., Kop'ev P.S., Alferov Zh.I., Bimberg D. Quantum dot heterostructures: fabrication, properties, lasers. // Fiz. Tehn. Pol., 1998, vol. 32, No. 4, p. 385-410 (in Russian).

\section{Спектр електрона в обмеженій циліндричній наногетеросистемі зі скінченною глибиною потенціальної ями}

\author{
В.А.Головацький, О.М.Войцехівська, М.Я.Міхальова, \\ М.М.Ткач \\ Чернівецький національний університет, \\ 274012 Чернівці, вул. Коцюбинського, 2 \\ Отримано 9 листопада 2000 р.
}

На основі варіаційного методу з варіаційним переметром у гамільтоніані розраховано електронний спектр у циліндричній квантовій точці HgS, розміщеній у середовищі ZnS. Встановлена залежність енергетичного спектру, розрахованого в рамках моделі безмежно глибокої потенціальної ями, задовільно працює лише для основного рівня і при досить великих розмірах квантової ями.

Ключові слова: електрон, квантова точка, нанокристал

PACS: $79.60 . j v$ 
